\title{
Avaliação crítica dos benefícios e limitações da ressonância magnética como método complementar no diagnóstico das malformações fetais*
}

\author{
A critical review of benefits and limitations of magnetic resonance imaging \\ as a complementary method in the diagnosis of fetal malformations \\ Renato Luis da Silveira Ximenes ${ }^{1}$, Jacob Szejnfeld ${ }^{2}$, Andréa Regina da Silveira Ximenes ${ }^{3}$, \\ Valdir Zanderigo ${ }^{4}$
}

Resumo OBJETIVO: Avaliar, por meio da ressonância magnética, uma série de fetos com diagnóstico ultra-sonográfico de malformação, a fim de estabelecer os benefícios e limites diagnósticos proporcionados pela técnica de ressonância magnética fetal, em comparação com a ultra-sonografia. MATERIAIS E MÉTODOS: Foram estudadas 40 mulheres entre 15-35 semanas de gestação com diagnóstico de anomalia fetal durante o exame de ultra-sonografia. As pacientes foram encaminhadas para o estudo complementar com ressonância magnética. As indicações para o estudo da ressonância magnética fetal foram: anomalias do sistema nervoso central, do tórax, do abdome, renais, esqueléticas e tumores. A avaliação pós-natal incluiu a revisão das imagens de ultra-sonografia e ressonância magnética, o acompanhamento do nascimento, exames laboratoriais, radiológicos e necropsia. RESULTADOS: Os resultados mostraram que os estudos complementares com ressonância magnética fetal trouxeram informações adicionais em $60 \%$ dos casos estudados. Os benefícios da ressonância magnética fetal foram: ampliação da avaliação global, aumento do campo de avaliação, maior resolução tecidual pelo uso de seqüências, e avaliação em pacientes obesas e com oligoidrâmnio. Os limites da ressonância magnética fetal foram: evitar exame no primeiro trimestre, avaliação do fluxo sanguíneo, movimentação fetal, claustrofobia materna, estudo do coração fetal e esqueleto. CONCLUSÃO: A ressonância magnética fetal pode ser utilizada como método complementar para a avaliação das malformações fetais. Unitermos: Imagem por ressonância magnética fetal; Ultra-sonografia; Malformações; Single-shot; HASTE; Diagnóstico pré-natal.

Abstract OBJECTIVE: The present study was aimed at evaluating by means of magnetic resonance imaging a series of fetuses with sonographic diagnosis of malformation, establishing the diagnostic benefits and limitations of fetal magnetic resonance imaging as compared with ultrasonography. MATERIALS AND METHODS: Forty women between 15-35 gestational weeks and previously diagnosed with fetal abnormality by ultrasonography were referred to undergo complementary fetal magnetic resonance imaging, particularly for evaluating abnormalities in the fetal central nervous system, thorax, abdomen, renal system, skeletal system, and tumors. The whole evaluation process included a review of the fetal ultrasonography and magnetic resonance images, postnatal follow-up, laboratory tests, imaging studies and necropsy. RESULTS: The present study has demonstrated that complementary magnetic resonance imaging did provide further information in $60 \%$ of cases, with the following benefits: improved information on the fetus as a whole, with a large field of view, higher anatomic resolution provided by fast sequences, superior soft tissue contrast resolution, besides the fact that the visualization of the fetus is not significantly affected by maternal obesity or oligohydramnios. Limitations of the method include contraindication in the first gestational trimester and in cases of maternal claustrophobia, sensitivity to fetal motion, low sensitivity for detecting cardiovascular and skeletal malformations. CONCLUSION: Fetal magnetic resonance imaging plays a significant role as a complementary method for the diagnosis of fetal anomalies.

Keywords: Fetal magnetic resonance imaging; Ultrasonography; Malformations; Single-shot; HASTE; Prenatal diagnosis.

Ximenes RLS, Szejnfeld J, Ximenes ARS, Zanderigo V. Avaliação crítica dos benefícios e limitações da ressonância magnética como método complementar no diagnóstico das malformações fetais. Radiol Bras. 2008;41(5):313-318.

* Trabalho realizado no Departamento de Diagnóstico por Imagem da Universidade Federal de São Paulo/Escola Paulista de Medicina (Unifesp/EPM), São Paulo, SP, no Cura - Diagnóstico e Imagem, São Paulo, SP, e no Centrus - Centro de Ultra-Sonografia e Medicina Fetal de Campinas, Campinas, SP, Brasil.

1. Pós-graduando do Departamento de Diagnóstico por Imagem da Universidade Federal de São Paulo/Escola Paulista de Medicina (Unifesp/EPM), São Paulo, SP, Diretor Científico do
Centrus - Centro de Ultra-Sonografia e Medicina Fetal de Campinas, Campinas, SP, Brasil.

2. Livre-Docente, Professor do Departamento de Diagnóstico por Imagem da Universidade Federal de São Paulo/Escola Paulista de Medicina (Unifesp/EPM), Diretor do Cura - Diagnóstico e Imagem, São Paulo, SP, Brasil.

3. Radiologista do Centrus - Centro de Ultra-Sonografia e Medicina Fetal de Campinas, Campinas, SP, Brasil.
4. Biomédico do Cura - Diagnóstico e Imagem, São Paulo, SP, Brasil.

Endereço para correspondência: Dr. Renato Ximenes. Centrus - Centro de Ultra-Sonografia e Medicina Fetal de Campinas. Avenida Francisco Glicério, 2132, Guanabara. Campinas, SP, Brasil, 13023-100. E-mail: rximenes@centrus.com.br

Recebido para publicação em 24/9/2007. Aceito, após revisão, em 12/11/2007. 


\section{INTRODUÇÃO}

O objetivo deste trabalho foi o de avaliar uma série de 40 fetos com diagnóstico ultra-sonográfico de malformação, realizando estudo complementar com ressonância magnética (RM), para estabelecer os benefícios e limites desta técnica, em comparação com a ultra-sonografia (US).

A US tem sido usada extensivamente, nas últimas quatro décadas, para averiguar as malformações fetais durante o pré-natal $^{(1)}$. Na avaliação obstétrica, a US constitui o método ideal para o diagnóstico por imagem, pois não emite radiação, não é invasivo e é relativamente barato. Por outro lado, apresenta algumas desvantagens referentes à experiência do operador, ao tipo de equipamento, ao campo limitado de avaliação, à obesidade materna, à redução acentuada do líquido amniótico, à posição fetal e a artefatos relacionados com o método (p. ex.: sombra acústica).

O emprego da RM para o estudo fetal foi descrito, pela primeira vez, em $1983^{(2)}$, e no início só se aplicava à avaliação da placenta e dos órgãos maternos ${ }^{(3)}$. Nessa época, o maior problema consistia na movimentação fetal durante a aquisição das imagens, ficando os estudos com RM restritos a avaliações volumétricas ${ }^{(4)}$. Para tentar eliminar a movimentação do feto, utilizaram-se drogas como os relaxantes musculares, por via indireta ${ }^{(5)}$ ou direta (cordocentese fetal) ${ }^{(6)}$.

Avanços recentes nos equipamento de RM incluíram a incorporação de protocolos de aquisição de imagens de forma ultra-rápida, quase eliminando, assim, os artefatos de movimentação fetal ${ }^{(7)}$. Essas técnicas de aquisição rápida, conhecidas como single-shot fast spin echo e half-Fourier acquisition turbo spin-echo (HASTE) formam protocolos cujo tempo de aquisição é menor que um segundo ${ }^{(8)}$.

A US é o método de rastreamento primário para o diagnóstico das anomalias fetais, podendo, ainda, ser utilizada como método complementar para os estudo das malformações fetais durante o pré-natal.

\section{MATERIAIS E MÉTODOS}

Por meio da RM, efetuaram-se estudos em pacientes entre 15-35 semanas de ges- tação com diagnóstico ultra-sonográfico de malformação fetal. O protocolo foi aprovado pelo Comitê de Ética da Instituição onde o trabalho foi realizado. Termo de consentimento livre e esclarecido foi assinado por todas as participantes, antes de sua inclusão no estudo.

Foram incluídas 40 pacientes com malformações fetais diagnosticadas durante o exame de US. Foram excluídas do estudo oito pacientes com sintomas de desconforto e/ou claustrofobia. As anomalias fetais foram divididas em subcategorias: sistema nervoso central (60\%), tórax (8\%), abdome $(8 \%)$, renal (10\%), esquelético (2\%), tumores fetais $(5 \%)$, gestação gemelar $(2 \%)$ e síndromes $(2 \%)$.

Obrigatoriamente, os casos incluídos tiveram acompanhamento pós-natal, que abrangia avaliação na sala de parto, exames de imagem e laboratoriais e, na ocorrência de óbito, realização de necropsia, quando autorizada pela família. A análise dos estudos com RM fetal foi revisada por dois radiologistas com experiência em medicina fetal. Esses casos somente foram incluídos neste estudo após sua análise completa e revisão sistemática das imagens de US, RM fetal e confirmação pós-natal.

Os estudos ultra-sonográficos foram executados com o auxílio de equipamentos HDI 4000 com transdutor broad band 4,07,0 MHz (Philips; Bothel, EUA) e Acuson Sequoia com transdutores multifreqüenciais 3,5-5,0 MHz e 4,0-8,0 MHz (Acuson; Mountain View, EUA). Adotou-se o exame ultra-sonográfico como método primário de rastreamento de anomalias fetais e como referência para indicação do estudo complementar por RM. O diagnóstico ultra-sonográfico foi estabelecido entre $15 \mathrm{e}$ 36 semanas de gestação (média de 28 semanas). Houve um intervalo máximo de 15 dias entre os exames de US e RM. Para os exames ultra-sonográficos utilizaram-se modo-B (escala de cinza), bidimensional (2D) e tridimensional (3D), ou, ainda, recursos como harmônica e Doppler colorido em casos selecionados. Todos os exames ecográficos foram armazenados em mídias digitais, incluindo todas as seqüências e planos (coronal, sagital e axial).

Os estudos fetais com a RM foram realizados em equipamentos de alto campo de 1.0 T Impact Magnetom System (Siemens
Medical Systems; Iselin, EUA) e de 1,5 T Sonata System (Siemens Medical Systems; Iselin, EUA), com bobina de corpo de alto campo.

Os protocolos de imagem da RM fetal incluíram sequiências ponderadas em $\mathrm{T} 1 \mathrm{e}$ $\mathrm{T} 2$ nos três planos ortogonais do corpo fetal. Iniciamos os estudos com sequiências ponderadas em T2, nos planos sagitais, coronais e axiais, utilizando cada uma como escanograma (scout) para a próxima sequiência de imagens. A sequiência mais empregada para os estudos de RM fetal é a T2 $\mathrm{HASTE}^{(7)}$. Esta seqüência é a preferida em estudos fetais, em virtude não apenas do excelente contraste dos tecidos fetais proporcionado pelas imagens ponderadas em $\mathrm{T} 2$, como também à alta relação sinal-ruído e relativa insensibilidade aos artefatos de suscetibilidade e movimento. As sequiências ponderadas em T2 HASTE proporcionam excelente contraste entre os tecidos fetais, limitando os artefatos relacionados a respiração materna e movimentos fetais ${ }^{(\boldsymbol{9})}$.

As imagens ponderadas em T1 são adotadas para a avaliação de produtos de sangue e de lesões com conteúdo de gordura, sendo realizadas como seqüências de gradiente de imagem gradient refocused acquisition in the steady state (GRASS) e fast slow angle shot (FLASH). As imagens obtidas nas aquisições em T1 são pouco mais degradadas devido à sua maior suscetibilidade à movimentação fetal durante $\mathrm{o}$ exame $^{(10)}$.

A maioria dos estudos sugere que a RM pode ser realizada durante a gestação ${ }^{(\mathbf{1 1}-13)}$. As preocupações referem-se à saúde materna e fetal. Os cuidados com a mãe são os mesmos dispensados às pacientes nãográvidas. A preocupação com o feto relaciona-se a teratogênese e danos acústicos. Neste estudo não utilizamos sedação materna ou contrastes.

\section{RESULTADOS}

Neste estudo comparativo entre a RM e a US para a avaliação das anomalias fetais houve correlação e comparação entre os resultados da RM e os achados ultra-sonográficos. Foi possível constatar que a RM fetal trouxe informações adicionais à US em 60\% dos casos (24/40) observados (Quadro 1). 
Quadro 1 Comparação entre US e RM, no caso de informações adicionais.

\begin{tabular}{|c|c|}
\hline US e RM: RM sem informação adicional & US e RM: RM com informação adicional \\
\hline $\begin{array}{l}\text { Hemorragia intracraniana grau IV } \\
\text { Malformação de Dandy-Walker } \\
\text { Válvula de uretra posterior } \\
\text { Ventriculomegalia borderline (Átrio posterior entre } \\
\text { 10-15 mm) } \\
\text { Gastrosquise } \\
\text { Anencefalia } \\
\text { Seqüência acrania-anencefalia } \\
\text { Síndrome de Body-Stalk } \\
\text { Artrogripose } \\
\text { Transfusão feto-fetal } \\
\text { Obstrução de vias aéreas superiores (OVAS) }\end{array}$ & $\begin{array}{l}\text { Aneurisma da veia de Galeno: classificação do tipo de malformação arteriovenosa, atraso na } \\
\text { formação dos sulcos } \\
\text { Arnold-Chiari tipo II: avaliação do grau de herniação do cerebelo, cortes sagitais da fossa } \\
\text { posterior e forame magno } \\
\text { Cisto aracnóide: localização e avaliação de possível compressão de estruturas adjacentes } \\
\text { Síndrome Perlmann: avaliação do parênquima renal } \\
\text { Teratoma sacrococcígeo: identificação da lesão pré-sacral } \\
\text { Teratoma cervical: limites e textura da massa, identificação da traquéia (compressão e desvio) } \\
\text { Encefalocele: tamanho e estruturas herniadas } \\
\text { Estenose da junção uretero-pélvica: local da estenose } \\
\text { Holoprosencefalia lobar: alteração do hipocampo e sulcos } \\
\text { Focos de hemorragia intra-hepática confirmados com seqüências em T1 } \\
\text { Síndrome de CHARGE: alterações da face (fenda palatina) } \\
\text { Holoprosencefalia alobar: cisto dorsal, hipocampo e compressão da fossa posterior }\end{array}$ \\
\hline
\end{tabular}

CHARGE: C, coloboma do olho; H, defeitos do coração; A, atresia de coanas; R, retardo do crescimento e/ou desenvolvimento; G, anomalias geniturinárias; E, anomalias da orelha e/ou auditivas.

Nos casos de anomalias do sistema nervoso central, a investigação com a RM fetal forneceu informações adicionais em 16/24 (57\%) dos casos. Como benefícios da RM no estudo do sistema nervoso central, obtivemos uma visão mais detalhada da formação de sulcos, do processo de formação das camadas e mielinização, do espaço subaracnóide, da ausência de artefatos ósseos, da obtenção de cortes nos três planos, e uma melhor definição tecidual e anatômica (Figura 1).

Nos casos de espinha bífida, a RM fetal trouxe informações adicionais na avaliação da fossa posterior, tendo sido avaliado o grau da herniação do cerebelo (deslocamento para baixo, compressão e hipoplasia do cerebelo) (Figura 2).

Neste estudo foram avaliados três casos de anomalias torácicas. No caso de hérnia diafragmática, a RM fetal forneceu informações adicionais na identificação do conteúdo e posição do fígado. No caso de obstrução das vias aéreas superiores, a RM não trouxe informações adicionais sobre as estruturas envolvidas no processo de obstrução e/ou etiologia.

Foram estudados três casos de anomalias do trato gastrintestinal. Estudamos um caso de gastrosquise, para o qual a RM fetal não trouxe informações adicionais.

Foram estudados quatro casos de anomalias do trato geniturinário. Como benefícios da RM fetal na avaliação das anomalias do trato geniturinário, observamos: identificação do ponto da estenose da jun-

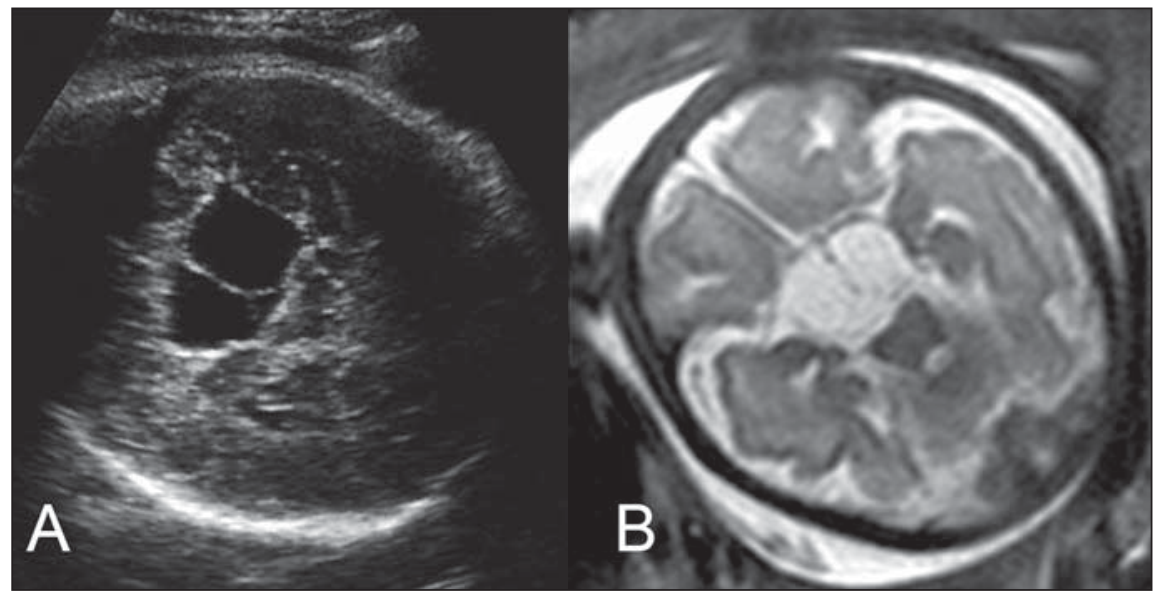

Figura 1. A: US, corte axial no nível do diâmetro biparietal. Visualização de estrutura cística. A sombra acústica impede a visualização completa da calota craniana. B: RM, seqüência ponderada em T2, mostra excelente contraste tecidual. A área com hipersinal corresponde a formação cística, compatível com cisto aracnóide.

ção uretero-pélvica sem a utilização de meios de contraste (Figura 3) e boa resolução tecidual mesmo nos casos de oligoidrâmnio e seqüências de difusão para avaliação da função renal. Como limitações, assinalamos a impossibilidade do diagnóstico precoce das anomalias do trato geniturinário.

Por meio da RM fetal investigamos um caso de alteração esquelética, não tendo sido obtidas informações adicionais na avaliação dos ossos longos. Não foi possível avaliar a movimentação das extremidades e articulações durante a realização do exame de RM.

Foram estudados dois casos de tumores fetais. No estudo das massas anteriores cervicais, os benefícios da RM estiveram dirigidos para o objetivo de estabelecer o diagnóstico diferencial. No caso de teratoma cervical, as imagens ponderadas em T2 possuem a capacidade de mostrar alta definição e contraste tecidual, exibindo detalhadamente as margens e a textura da massa (Figura 4). Na avaliação do tumor sacrococcígeo, a RM traz benefícios relacionados com o tamanho, a extensão do tumor e o comprometimento de estruturas adjacentes $^{(\mathbf{1 4})}$. Na avaliação dos tumores fetais, a limitação da RM fetal consistiu na incapacidade de demonstrar a atividade vascular.

Foi estudado um caso de gestação gemelar monocoriônica-diamniótica que tinha 


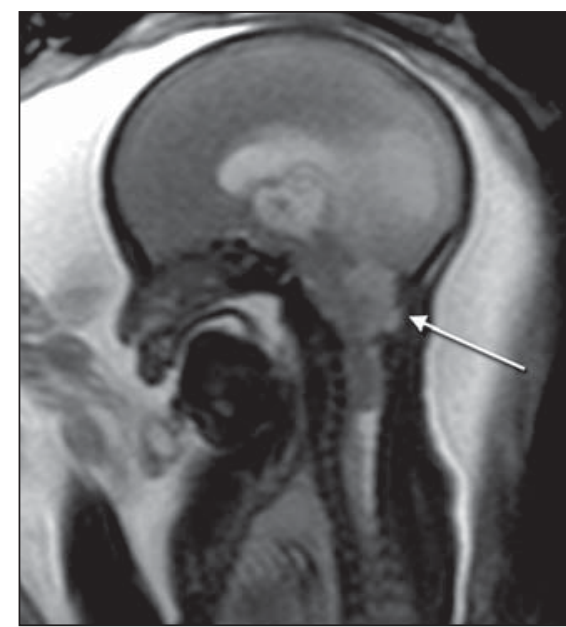

Figura 2. RM fetal, seqüência ponderada em T2 corte sagital, mostrando o quarto ventrículo extremamente baixo e estreito, abaixo do forame magno. Os hemisférios cerebelares estão comprimidos (hipoplásicos).

diagnóstico ultra-sonográfico de síndrome da transfusão feto-fetal. Não obtivemos informações adicionais no estudo com a RM fetal.

As imagens da US e da RM fetal foram comparadas, sendo avaliados os pontos benéficos e as limitações entre estes métodos no diagnóstico pré-natal das anomalias anatômicas. No Quadro 2 podemos observar as limitações da US e da RM no diag-

Quadro 2 Limitações da US e da RM fetal no diagnóstico pré-natal.

\begin{tabular}{|ll|}
\hline \multicolumn{2}{|c|}{ US } \\
\hline Obesidade materna & Alto custo do exame \\
Oligoidrâmnio acentuado & Dificuldade na padronização dos cortes \\
Campo diagnóstico limitado & Movimentação fetal \\
Resolução tecidual inferior & Claustrofobia \\
Visualização da anatomia fetal no terceiro tri- & Movimentação fetal \\
mestre & Artefatos: movimentação fetal \\
Artefatos: sombra posterior & Não deve ser realizada no primeiro trimestre \\
Obtenção de três planos pode ser prejudicada & \\
por artefatos de sombra acústica posterior & \\
\hline
\end{tabular}

Quadro 3 Benefícios ou pontos positivos da US e da RM fetal no diagnóstico pré-natal.

\begin{tabular}{|ll|}
\hline \multicolumn{1}{|c|}{ US } & \multicolumn{1}{c|}{ RM fetal } \\
\hline Baixo custo & Amplo campo diagnóstico \\
Método em tempo real & Alta resolução tecidual \\
Padronização estabelecida dos cortes anatômi- & Identificação de imagens compatíveis com \\
cos & hemorragias ou gordura nas seqüências em \\
Mapeamento com Doppler colorido & T1 \\
Quantificação dos fluxos vasculares com Dop- & Obtenção de três planos ortogonais sem arte- \\
pler & fatos \\
Pode ser utilizada no primeiro trimestre & Utilização de seqüências que avaliam a fun- \\
& ção renal \\
\hline
\end{tabular}

Figura 3. RM fetal, seqüência ponderada em T2 e corte parassagital oblíquo, mostrando dilatação da pelve renal e o ponto de estenose no ureter proximal (seta).

nóstico pré-natal. O ponto favorável e comum entre os métodos é a não-utilização de radiação ionizante. No Quadro 3 podemos observar os benefícios da US e da RM no diagnóstico pré-natal.

\section{DISCUSSÃO}

A US representa o método de escolha para o rastreamento primário das malfor-

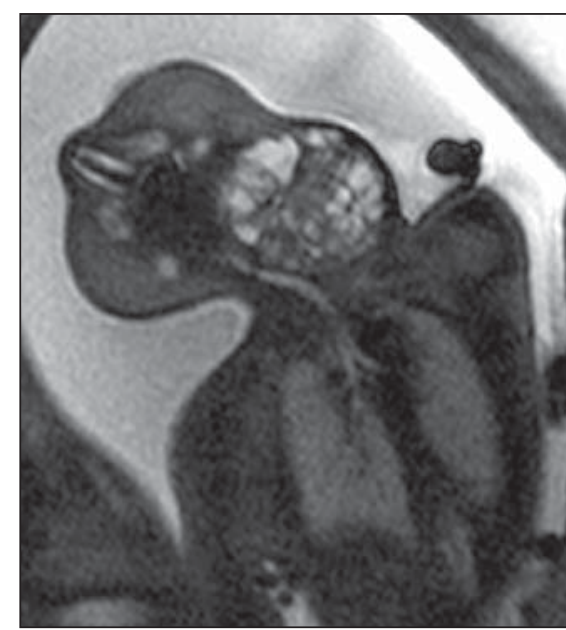

Figura 4. RM em corte axial oblíquo mostrando massa cenvical com margens definidas e o "efeitomassa" comprimindo e deslocando a traquéia.

mações fetais durante o pré-natal, em virtude do seu baixo custo operacional e da sua capacidade de ser em tempo real. Entretanto, pudemos verificar que a RM fetal pode fornecer informações adicionais aos estudos com US. À medida que a experiência com a RM fetal aumentar, poderemos incluir um número maior de outras indicações para o estudo complementar com RM.

Pesquisas mostram que a RM fetal pode avaliar as mudanças seqüenciais do desenvolvimento do sistema nervoso central, avaliar com maior acurácia as paredes dos ventrículos e espaços subaracnóides, demonstrando, particularmente, a organização do tecido intraparênquima devido à excelente resolução de contraste nas seqüências ponderadas em $\mathrm{T} 2^{(\mathbf{1 5 - 1 7 )}}$. Nota-se como limitação, nos estudos do sistema nervoso central, que a avaliação da anatomia intracraniana tem qualidade inferior nos casos acima de 20 semanas, podendo estar relacionada com o tamanho reduzido dos órgãos a serem avaliados ${ }^{(\mathbf{1 8})}$. O acompanhamento por RM fetal no terceiro trimestre deve ser realizado uma ou duas semanas antes do parto ${ }^{(\mathbf{1 9})}$.

É consenso geral que o estudo das malformações fetais do sistema nervoso central pela RM tornou-se um método com valor diagnóstico confiável e seguro ${ }^{(\mathbf{1 6})}$. Já na avaliação do sistema nervoso central, a US apresenta limitações relacionadas com artefatos produzidos pela calota craniana e 
ossos (produção de sombra acústica posterior), idade gestacional, posição fetal, planos de estudo e obesidade materna. A US pode avaliar o fluxo vascular de estruturas intracranianas e cardíacas durante a gestação, fornecendo informações importantes da hemodinâmica fetal.

As vantagens da RM na avaliação dos fetos com anomalias do tórax têm sido expostas na literatura em vários $\operatorname{artigos}^{(\mathbf{2 0})}$. Até o momento, não observamos benefícios na utilização da RM fetal no estudo do coração fetal. O estudo com RM fetal pode ser útil no sentido de avaliar a intensidade do sinal nos pulmões, pois alguns trabalhos indicaram uma relação entre a intensidade desse sinal com a caracterização da hipoplasia pulmonar ${ }^{(\mathbf{2 1})}$. O cálculo volumétrico pulmonar tem-se mostrado um marcador melhor para a avaliação da hipoplasia pulmonar do que a análise do sinal ${ }^{(22,23)}$. Nos casos de hérnia diafragmática congênita, a localização do lobo esquerdo do fígado é um fator extremamente relevante no aconselhamento do prognóstico dos resultados perinatais, já que existe uma relação entre "fígado para cima" e "fígado para baixo" e a taxa de mortalidade perinatal, de $57 \%$ e 7\%, respectivamente ${ }^{(\mathbf{2 4})}$.

$\mathrm{O}$ estudo das doenças abdominais por RM varia de acordo com a idade gestacional e a capacidade de visualizar e diferenciar os diferentes órgãos do abdome. Estes, por sua vez, são dependentes do tamanho da estrutura, que faz variar o sinal característico da estrutura anatômica, dependente da seqüência utilizada. $\mathrm{O}$ conhecimento do desenvolvimento normal é fundamental para a interpretação das imagens de RM fetal. Enquanto nos adultos, para as informações obtidas nos estudos por RM, há a necessidade da utilização de meio de contraste, no período intra-uterino empregamos um contraste natural, o "líquido amniótico”, e em alguns casos, o conteúdo intestinal como o mecônio. Existe uma variação na presença desse meio de contraste natural, de acordo com a idade gestacional, provocando diferentes aspectos imaginológicos. O intestino delgado pode ser identificado com sequiências ponderadas em T1, com sinal hiperintenso semelhante ao sinal do fígado. A RM fetal pode localizar o ponto correto da obstrução intestinal e também demonstrar atresias múltiplas ${ }^{(25)}$.
Como benefícios podemos destacar diagnósticos como atresia anal, em que se podem identificar enchimento do reto, malformações da cloaca, sinal hipointenso em $\mathrm{T} 1^{(\mathbf{2 6})}$. Nos casos de megacólon congênito (doença de Hirschsprung), em que o reto dilatado pode ser identificado com sinal de média intensidade na seqüência ponderada em $\mathrm{T} 2^{(27)}$, e nos casos de doenças da adrenal, a RM pode demonstrar melhor o diagnóstico diferencial entre neuroblastoma e os casos de hemorragia adrenal, sequiestro subdiafragmático, cistos renais $^{(\mathbf{2 8})}$ e linfangioma retroperitoneal $^{(29)}$. Como limitações nos casos de anomalias do abdome e gastrintestinais, podemos citar o diagnóstico precoce dos defeitos da parede abdominal (abaixo de 18 semanas) e a motilidade intestinal.

A US é um método excelente na avaliação das anomalias do trato geniturinário, no entanto, em casos de obesidade materna e oligoidrâmnio, pode ter limitações no estudo detalhado do trato geniturinário ${ }^{(30)}$.

No estudo de anomalias esqueléticas, as limitações da RM estão relacionadas com a impossibilidade do estudo da movimentação fetal, a dificuldade da obtenção de planos corretos para a avaliação do esqueleto, a impossibilidade de se fazer a reconstrução 3D do esqueleto (como podemos fazer na tomografia computadorizada) e a impossibilidade de mensurar o grau de calcificação.

No diagnóstico pré-natal das anomalias fetais, os limites e benefícios de ambos os métodos causaram a impressão de que se deve complementar a US com a RM fetal em casos selecionados. É importante frisar que a movimentação fetal é ainda um fator limitante, uma vez que a aquisição de planos anatômicos padronizados e simétricos fica pouco reprodutível.

\section{CONCLUSÃO}

No presente trabalho analisaram-se, comparativamente, a US e a RM para a investigação das malformações fetais, no diagnóstico pré-natal. Além disso, estabeleceram-se os limites e benefícios da RM como estudo complementar. A US é o método de rastreamento primário e foi observado que a RM fetal fornece informações adicionais.
Podemos concluir que a RM pode ser utilizada como método complementar à US para o diagnóstico de malformações fetais.

\section{REFERÊNCIAS}

1. Nicolaides KH, Snijders RJ, Gosden CM, et al Ultrasonographically detectable markers of fetal chromosomal abnormalities. Lancet. 1992;340: 704-7.

2. Smith FW, Adam AH, Phillips WD. NMR imaging in pregnancy. Lancet. 1983;1:61-2.

3. Smith FW. The potential use of nuclear magnetic resonance imaging in pregnancy. J Perinat Med. 1985;13:265-76.

4. Weinreb JC, Lowe TW, Santos-Ramos R, et al. Magnetic resonance imaging in obstetric diagnosis. Radiology. 1985;154:157-61.

5. Levine D, Barnes PD, Sher S, et al. Fetal fast MR imaging: reproducibility, technical quality, and conspicuity of anatomy. Radiology. 1998;206: 549-54.

6. Seeds JW, Corke BC, Spielman FJ. Prevention of fetal movement during invasive procedures with pancuronium bromide. Am J Obstet Gynecol. 1986;155:818-9

7. Levine D, Barnes PD, Edelman RR. Obstetric MR imaging. Radiology. 1999;211:609-17.

8. Levine D, Hatabu H, Gaa J, et al. Fetal anatomy revealed with fast MR sequences. AJR Am J Roentgenol. 1996;167:905-8.

9. Yamashita Y, Namimoto T, Abe Y, et al. MR imaging of the fetus by a HASTE sequence. AJR Am J Roentgenol. 1997;168:513-9.

10. Bradley WG Jr. MR appearance of hemorrhage in the brain. Radiology. 1993;189:15-26.

11. Formica D, Silvestri S. Biological effects of exposure to magnetic resonance imaging: an overview. Biomed Eng Online. 2004;3:11. [Acessado em: 3/10/2007]. Disponível em: http://www. biomedical-engineering-online.com/content/3/1/ 11

12. Beers GJ. Biological effects of weak electromagnetic fields from $0 \mathrm{~Hz}$ to $200 \mathrm{MHz}$ : a survey of the literature with special emphasis on possible magnetic resonance effects. Magn Reson Imaging. 1989;7:309-31.

13. Budinger TF. MR safety: past, present, and future from a historical perspective. Magn Reson Imaging Clin N Am. 1998;6:701-14.

14. Avni FE, Guibaud L, Robert Y, et al. MR imaging of fetal sacrococcygeal teratoma: diagnosis and assessment. AJR Am J Roentgenol. 2002; 178:179-83.

15. Lan LM, Yamashita Y, Tang Y, et al. Normal fetal brain development: MR imaging with a half-Fourier rapid acquisition with relaxation enhancement sequence. Radiology. 2000;215:205-10.

16. Levine D, Barnes PD, Robertson RR, et al. Fast MR imaging of fetal central nervous system abnormalities. Radiology. 2003;229:51-61.

17. Levine D, Trop I, Mehta TS, et al. MR imaging appearance of fetal cerebral ventricular morphology. Radiology. 2002;223:652-60.

18. Levine D, Barnes PD, Madsen JR, et al. Central nervous system abnormalities assessed with prenatal magnetic resonance imaging. Obstet Gynecol. 1999;94:1011-9.

19. Robertson R, Caruso PA, Truwit CL, et al. Disor- 
ders of brain development. In: Atlas SW, editor Magnetic resonance imaging of the brain and spine. 3rd ed. Philadelphia: Lippincot Williams \& Wilkins; 2002. p. 279-370.

20. Levine D, Barnewolt CE, Mehta TS, et al. Fetal thoracic abnormalities: MR imaging. Radiology. 2003;228:379-88.

21. Ikeda K, Hokuto I, Tokieda K, et al. A congenital anterior diaphragmatic hernia with massive pericardial effusion requiring neither emergency pericardiocentesis nor operation. A case report and review of the literature. J Perinat Med. 2002; 30:336-40.

22. Paek BW, Coakley FV, Lu Y, et al. Congenital diaphragmatic hernia: prenatal evaluation with MR lung volumetry - preliminary experience. Radiology. 2001;220:63-7.
23. Coakley FV, Lopoo JB, Lu Y, et al. Normal and hypoplastic fetal lungs: volumetric assessment with prenatal single-shot rapid acquisition with relaxation enhancement MR imaging. Radiology. 2000;216:107-11.

24. Leung JW, Coakley FV, Hricak H, et al. Prenatal MR imaging of congenital diaphragmatic hernia. AJR Am J Roentgenol. 2000;174:1607-12.

25. Benachi A, Sonigo P, Jouannic JM, et al. Determination of the anatomical location of an antenatal intestinal occlusion by magnetic resonance imaging. Ultrasound Obstet Gynecol. 2001;18: 163-5.

26. Saguintaah M, Couture A, Veyrac C, et al. MRI of the fetal gastrointestinal tract. Pediatr Radiol. 2002;32:395-404.

27. Ohgiya Y, Gokan T, Hamamizu K, et al. Fast MRI in obstetric diagnoses. J Comput Assist Tomogr. 2001;25:190-200.

28. Aslan H, Ozseker B, Gul A. Prenatal sonographic and magnetic resonance imaging diagnosis of cystic neuroblastoma. Ultrasound Obstet Gynecol. 2004;24:693-4.

29. Houlihan C, Jampolsky M, Shilad A, et al. Prenatal diagnosis of neuroblastoma with sonography and magnetic resonance imaging. J Ultrasound Med. 2004;23:547-50.

30. Levine D, Goldstein RB, Callen PW, et al. The effect of oligohydramnios on detection of fetal anomalies with sonography. AJR Am J Roentgenol. 1997;168:1609-11. 